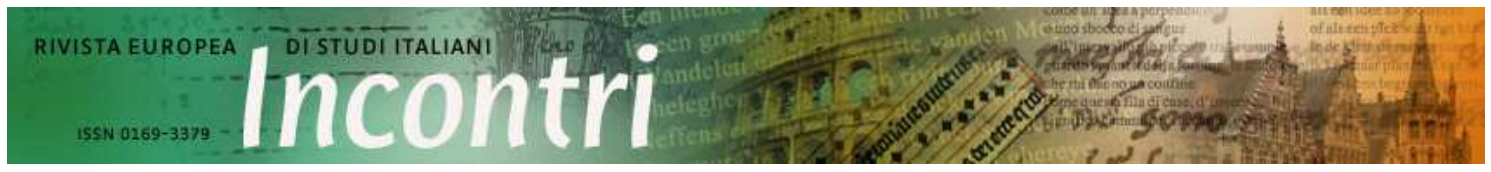

URN:NBN:NL:UI:10-1-101360 - Publisher: Igitur publishing

Content is licensed under a Creative Commons Attribution 3.0 License

Anno 26, 2011 / Fascicolo 2 - Website: www.rivista-incontri.nl

\title{
La ricezione della Shoah in Italia: una memoria aperta
}

\author{
Recensione di: Emiliano Perra, Conflicts of Memory. The Reception \\ of Holocaust Films and TV Programmes in Italy, 1945 to the \\ Present, Peter Lang, 'Italian Modernities' vol. 8, Oxford-Bern, \\ 2010. ISBN: 9783039118809.
}

\section{Natalie Dupré e Monica Jansen}

Il titolo del volume, in italiano 'Conflitti di memoria. La ricezione in Italia di film e programmi televisivi sulla Shoah dal 1945 al presente', nella sua precisione evidenzia in pieno le qualità critiche e oggettive dello studio di Emiliano Perra, visiting fellow al dipartimento di Studi Storici dell'Università di Bristol. Avendo un duplice scopo, la monografia di Perra compie un'acuta disamina delle interpretazioni conflittuali e politicizzate delle narrazioni sulla Shoah, le quali hanno ostacolato l'elaborazione di una memoria collettiva del ruolo svolto dall'Italia nella persecuzione degli ebrei. Nello stesso tempo Perra analizza la ricezione dei film e programmi televisivi dedicati alla Shoah, adottando un metodo 'meta-interpretativo' (p. 23); i film e le trasmissioni presi in esame da Perra risultano rilevanti non tanto per i loro contenuti o per i punti di vista dei singoli registi, quanto per il fatto che fanno trasparire una 'sum of meanings' (p. 23). Da questa somma di opinioni emerge una memoria della Shoah dominata da una metanarrazione volta all'innocenza e al vittimismo. I singoli casi di ricezione, presentati sia in ordine cronologico che per attinenza tematica, dimostrano come il trauma collettivo della guerra continua ad attanagliare l'Italia, paese che, secondo Perra, non sembra in grado di convertire la memoria storica in un sistema di valori che possa dare una direzione a scelte presenti e future (p. 231). In questo senso il saggio aspira non solo a compiere una ricostruzione storica, ma anche a tracciare i principi di un'etica e di una politica riguardo alla memoria della Shoah in Italia.

Risalendo agli anni Quaranta e Cinquanta, Perra dimostra come nel dopoguerra la ricezione di film popolari sulla Shoah - tra cui primeggia il 
melodramma della Resistenza L'ebreo errante (1947-48) - è calata interamente nei paradigmi del cattolicesimo e dell'etica della Resistenza, e dunque tende a esimere gli italiani dalla loro colpa nella persecuzione degli ebrei. In seguito Perra indica come la ripresa nei primi anni Sessanta del mito della Resistenza in un contesto fortemente condizionato dalla Guerra Fredda ostacolò non solo un possibile ripensamento critico del coinvolgimento dell'Italia nella Shoah, ma anche più semplicemente l'avvio di un processo di riconoscimento della specificità storica dell'evento, che altri paesi come gli USA e la Germania Ovest avevano iniziato a partire dal processo Eichmann. Un film di successo dell'epoca come Kapò (1960) che esplora, a partire dalla vicenda di un'adolescente ebrea, il complesso tema dell'impatto del sistema concentrazionario sulle vittime, all'epoca è percepito prima di tutto come un dramma sulla Resistenza. Negli anni Settanta invece è il cosiddetto 'nuovo discorso' nel cinema a denunciare il chiaroscuro del fascismo, discorso che somiglia negli intenti a ciò che Primo Levi teorizza negli stessi anni come la 'zona grigia'. La Shoah diventa così una metafora di un'ambivalenza morale non specificamente legata al contesto storico italiano, metafora ulteriormente complicata dai conflitti in Medio-Oriente che dividono in quel periodo la sinistra italiana. Negli anni Settanta la tendenza all'universalizzazione della Shoah, spesso in termini di perversione capitalista e sessuale, blocca il processo che potrebbe portare l'Italia al riconoscimento di una sua colpa nella persecuzione degli ebrei. Lina Wertmüller in Pasqualino Settebellezze, film uscito nel 1975, adotta il genere della commedia per raccontare la storia di un sopravvissuto ambiguo, soldato italiano dell'esercito fascista rappresentato appunto come antieroe e vittima di circostanza. Nemmeno con la trasmissione della miniserie americana Holocaust su Rete 1 nel 1979 cambiano i parametri dell'interpretazione; anzi, la ricezione della miniserie, giocata spesso sul contrasto tra la Germania nazista e l'Italia, punta a rafforzare maggiormente l'immagine degli 'italiani brava gente'.

Solo a partire dalla seconda metà degli anni Ottanta il dibattito sulla Shoah coinvolge il pubblico e gli storici italiani. Il mito antifascista deve cedere progressivamente a favore di un'attenzione più esclusiva per la Shoah che diventa la nuova chiave di lettura della guerra. Perra addirittura definisce il 1997, anno in cui esce La vita è bella di Benigni, come l'anno italiano della Shoah (p. 150). Nonostante la partecipazione italiana alla persecuzione e distruzione degli ebrei sia ormai unanimamente riconosciuta e commemorata in quanto crimine fascista (p. 152), in alcune pratiche di memoria selettiva rilevate da Perra si manifestano tuttora rivendicazioni revisioniste politicamente motivate (p. 186). Un capitolo a parte è inoltre dedicato alla visualizzazione del ruolo svolto da Papa Pio XII e dal Vaticano nello sterminio degli ebrei. Attraverso l'analisi della ricezione dello spettacolo teatrale Il Vicario di Hochhuth, e dei film Rappresaglia diretto da Pan Cosmatos e Amen. di Costa-Gravas, il dibattito italiano viene ricostruito in una prospettiva transnazionale e ricollegato alla creazione di una serie di nuovi eroi 'post-ideologici' della Shoah (p. 216), come ad esempio il famoso protagonista di Schindler's List. Tra questi eroi post-ideologici vi è senza dubbio anche Giorgio Perlasca, fascista salvatore di ebrei e 'Giusto tra le Nazioni' incarnato sullo schermo televisivo da Luca Zingaretti nella miniserie Perlasca: un eroe italiano, trasmesso nel 2002 durante il 
Giorno della Memoria con una audience di 11 milioni la prima sera e 13 milioni la seconda.

Limitando la sua analisi alla ricezione di film e programmi televisivi, Perra riesce a cogliere la storia della memoria collettiva italiana della Shoah nel pieno del dibattito pubblico e quotidiano. Anche se con il suo studio non ottiene risultati molto diversi rispetto alla letteratura esistente in materia, Perra conferisce maggiore coerenza al complesso lavoro di contestualizzazione centrandolo sulle due narrazioni di innocenza e vittimismo che per molti anni hanno dominato la memoria pubblica italiana. A p. 186 Perra afferma che 'fully investigating Italian responsibility in the Holocaust represents a great opportunity for the country to do justice not only to the victims, but also to its own memory' (p. 186). Lasciando aperta la risposta alla domanda di come concretamente l'Italia possa cogliere questa opportunità, Perra rileva con la sua nota etica che il dibattito sulla Shoah in Italia è sempre aperto, sollecitando il dibattito pubblico su questo e altri problematici nodi della memoria storica italiana.

\section{Natalie Dupré}

Hogeschool-Universiteit Brussel, Warmoesberg 26, 1000 Brussel, Belgio natalie.dupre@hubrussel.be

\section{Monica Jansen}

Universiteit Utrecht, Departement Moderne Talen - Sectie Italiaans, Trans 10, 3512 JK Utrecht

m.m.jansen@uu.nl 